\title{
BMJ Open South Australian prospective cohort study evaluating outcomes of maternal kidney and cardiac disease in pregnancy: a protocol
}

\author{
Jarrad Hopkins (D) , ${ }^{1}$ Erandi Hewawasam, ${ }^{1,2}$ Emily Aldridge, ${ }^{1}$ Prabha Andraweera, ${ }^{3}$ \\ Shilpanjali Jesudason, ${ }^{1,4}$ Margaret Arstall ${ }^{1}$
}

To cite: Hopkins J, Hewawasam E, Aldridge E, et al. South Australian prospective cohort study evaluating outcomes of maternal kidney and cardiac disease in pregnancy: a protocol. BMJ Open 2022;12:e059160. doi:10.1136/ bmjopen-2021-059160

- Prepublication history for this paper is available online. To view these files, please visit the journal online (http://dx.doi. org/10.1136/bmjopen-2021 059160).

Received 10 November 2021 Accepted 20 January 2022

Check for updates

(C) Author(s) (or their employer(s)) 2022. Re-use permitted under CC BY-NC. No commercial re-use. See rights and permissions. Published by BMJ.

${ }^{1}$ Faculty of Health and Medical Sciences, The University of Adelaide, Adelaide, South Australia, Australia

${ }^{2}$ Australia and New Zealand Dialysis and Transplant Registry, Adelaide, South Australia,

Australia

${ }^{3}$ Obstetrics and Gynaecology, University of Adelaide School of Medicine, Adelaide, South Australia, Australia

${ }^{4}$ Central and Northern Adelaide Renal and Transplantation Services (CNARTS), Royal Adelaide Hospital, Adelaide, South Australia, Australia

Correspondence to Dr Jarrad Hopkins; jarrad.hopkins@sa.gov.au

\section{ABSTRACT}

Introduction Chronic kidney disease (CKD) and cardiac disease are two significant health conditions that can impact a women's pregnancy; however, little is known about their prevalence and health impact within the population. These pregnancies are associated with significant risks of morbidity and mortality and propose a challenge to clinicians. The aim of this longitudinal cohort study is to prospectively record the incidence, prevalence, aetiology, outcomes and follow-up of maternal CKD and cardiac disease in the obstetric population of South Australia.

Methods and analysis This study is a state-wide multicentre prospective cohort study in South Australia that will begin recruitment in 2022 and is planned for at least 5 years. Pregnant women with chronic or acquired kidney or cardiac disease will be enrolled across the state's major public obstetric hospitals. The data collected will focus on the chronic disease aetiology, peripartum interventions, delivery, obstetric and neonatal outcomes, progression of underlying disease and patient-related outcome measures. Women will have data collected each trimester during pregnancy and then at follow-up 6 weeks, 6 months and 12 months post partum. Clear inclusion and exclusion criteria have been developed which importantly includes new diagnosis of chronic disease in pregnancy. Ethics and dissemination Approval was obtained from the local Health Network Human Research Ethics Committee. Summary data will be reviewed and reported in accordance with Strengthening the Reporting of Observational Studies in Epidemiology criteria 6 monthly and results will be published in peer-reviewed journals and presented at conferences. Findings will be presented to relevant local clinicians and hospitals at regular intervals. Consumer versions of research outputs will be developed in conjunction with the consumer reference group.

\section{INTRODUCTION}

Maternal conditions, including chronic and acquired conditions, can seriously affect maternal and fetal health and outcomes. Chronic kidney disease (CKD) and cardiac disease are two significant diseases that can influence a woman's pregnancy, deeming them to be at higher risk that women without
Strengths and limitations of this study

- This will be the only prospective, cohort study in Australia with a primary purpose of collecting and evaluating the impact of chronic kidney disease (CKD) or cardiac disease in pregnancy, which is an essential step towards addressing this paucity of knowledge.

- Existing pregnancy datasets/registries are not inclusive of early pregnancy loss or early stage disease; thus, this protocol is mandated.

- One of the highly novel aspects of this study is the parental self-reported follow-up of infant health post partum.

- This dataset will only collect pregnancies initially from public hospitals which may limit its completeness.

- Not all CKD and cardiac disease will be identifiable in pregnancy; hence, this study focuses on clearly defined cardiac disease and kidney disease revealed by early proteinuria or kidney function impairment.

a chronic or acquired condition. There are many complications arising from CKD and cardiac disease that may increase the risk of adverse pregnancy and fetal outcomes. This high-risk group adds disproportionately to the burden of serious maternal obstetric and neonatal morbidity. ${ }^{12}$ This protocol outlines the methodology of a statewide cohort study to capture this complex dataset and research incidence, prevalence, aetiology, outcomes and follow-up of maternal CKD and cardiac disease in the obstetric population. The study will provide answers to key questions about the impact of these diseases on pregnancy for the mother and the child as well as maternal kidney and cardiac function. Future models of risk stratification in pregnant women with complex kidney and cardiac comorbidities will be developed. 


\section{CKD and pregnancy}

CKD is an increasing contributor to morbidity and mortality that is often under-recognised in the community, disproportionately affecting Aboriginal and Torres Strait Islander and lower socioeconomic populations. ${ }^{34}$ It is a broad condition that describes the reduction in estimated glomerular filtration rate (eGFR) and/or albuminuria lasting at least 3 months and categorised into stages of severity from stage 1 to $5 .{ }^{5}$ CKD affects 1 in 10 Australians with $5 \%-6 \%$ of these people within reproductive age (18-44 years). ${ }^{6}$ In Australia, $1.2 \%$ of total burden of disease is a direct result of CKD and this percentage has been increasing overtime. ${ }^{3} 7$ CKD is also associated with $11 \%$ of all deaths recorded in Australia in 2017, signifying it's health impacts. ${ }^{4} 6$

The Australia and New Zealand Dialysis and Transplant Registry (ANZDATA) collects detailed information from all patients undergoing dialysis or those who have received a kidney transplant. Within this data set, a substantial percentage of women in Australia in 2020 on kidney replacement therapy (dialysis or kidney transplant) are of reproductive age; $25 \%$ of all women with a functioning kidney transplant and $51 \%$ of all women with kidney failure. ${ }^{3}$ Epidemiological studies have shown that the pregnancy rate for those receiving long-term dialysis is 5.8 in 1000 people per year and 21.4 in 1000 people per year for those with renal transplants. ${ }^{389}$ In total, this equates to $<50$ pregnancies per year in Australia. ${ }^{8}$ However, the ANZDATA data represent an underrepresentation of the burden of CKD in pregnancy as it only records patients with kidney failure receiving kidney replacement therapy and only collects limited obstetric and perinatal data. Existing perinatal datasets in Australia almost never capture kidney function as they are not routinely measured or recorded through pregnancy. Linkage of perinatal datasets and ANZDATA has been completed and will add to the body of knowledge about pregnancy in women with kidney failure, ${ }^{9}$ but this does not address earlier CKD stages.

It is difficult to report robust data on CKD in pregnant cohorts, due to challenges in diagnosing and capturing CKD in population datasets. Over 5\% of Australian women of childbearing age were found to have indicators of CKD in the form of albuminuria or abnormal GFR on a retrospective analysis. ${ }^{4}$ Internationally, the Norwegian HUNT II study, a population-level registry found indicators of $\mathrm{CKD}$ in $3.3 \%$ of women in their reproductive age. ${ }^{10}$ This was validated in an Italian cohort where $3 \%$ of pregnancies were complicated by CKD. ${ }^{11}$ In Australia, there is a paucity of knowledge regarding the incidence, prevalence and impact of all stages of CKD in pregnancy. This paucity is exaggerated due to the varying definitions and dynamic approach to measuring kidney disease in pregnant cohorts. ${ }^{12}$ Currently in Australia, all jurisdictions have a mandatory perinatal data collection for births $>20$ weeks' gestation. This captures a range of antenatal, obstetric and infant data but limited diseasespecific data, and no laboratory data. Exploration of the perinatal dataset in South Australia over 20 years revealed $0.3 \%$ of women had a diagnostic code for a kidney condition ascribed to them in pregnancy, ${ }^{13}$ but CKD stage was not known.

The prevalence of CKD within the Australian pregnant population is potentially rising due to the increase in diabetes, obesity and the increasing median age of women who become pregnant. ${ }^{614}$ Earlier stages of CKD are more prevalent and impose important risks, ${ }^{8}$ but remain poorly defined in Australia. International cohorts have described that even CKD stages 1 and 2 with the following additional risk factors resulted in poorer obstetric outcomes: significant proteinuria (300 mg/24 hours), hypertension, systemic diseases (such as lupus) or previous adverse obstetric history. ${ }^{15}$ The implications of $\mathrm{CKD}$ in pregnancy have been investigated and there is an association with worse obstetric and perinatal outcomes including preterm birth, small for gestational age, and pre-eclampsia and gestational hypertension. ${ }^{16}$ Interesting, even patients with stage $1 \mathrm{CKD}$, with no hypertension or proteinuria have increased risks of adverse pregnancy outcomes. $^{2}$ One South Australian study looking at ICD codes alone found that women with any kidney disease were 2.2 times more likely to have pregnancy induced hypertension, 1.3 times more likely to require caesarean section, 2.8 chance of preterm birth and 2.6 increased risk of the neonate requiring admission to intensive care. ${ }^{13} \mathrm{~A}$ previous systematic review and meta-analysis published in 2015 by Zhang et $a l^{12}$ support that early stages of CKD are implicated in worse pregnancy-related outcomes. Furthermore, one study found that approximately $8 \%$ of women with CKD stage 1 in pregnancy experienced a worsening of kidney function that caused them to shift their CKD staging or more severely commence kidney replacement therapy during pregnancy. ${ }^{17}$ This increased to $20 \%$ for women with a more severe baseline of kidney impairment in stages 4 and 5 , and in this cohort a permanent deterioration was observed in $31 \%$ of cases. ${ }^{18}$ Complications in pregnancy such as pre-eclampsia can have significant implications on CKD, one study showed that pre-eclampsia confers a 4.3 relative risk of subsequently developing end-stage kidney failure. ${ }^{19}$

\section{Cardiovascular disease and pregnancy}

Cardiovascular disease affects an estimated $4.8 \%$ of Australian women over the age of 18 and accounts for $12 \%$ of total burden of disease. ${ }^{4}$ The incidence, prevalence and hospitalisation rates of cardiovascular disease are rising in the 25-34 and 35-44 age brackets by $11 \%$ and $4.7 \%$, respectively. ${ }^{40}$ Worldwide, maternal cardiac disease is present between $1 \%$ and $4 \%$ of pregnancies and unfortunately accounts for $15 \%$ of maternal mortality. ${ }^{21}$ Of all obstetric Intensive care unit (ICU) admissions, $15 \%$ are the result of maternal cardiac disease. ${ }^{22}$ The number of women who have a pre-existing cardiovascular disease or develop cardiac problems during pregnancy is increasing. ${ }^{23}$ The significant physiological and haemodynamic changes of pregnancy can result in exacerbation 
or reveal heart disease that may not have been diagnosed. ${ }^{24}$ Women of childbearing age are vastly underrepresented in the field of cardiovascular research and often completely excluded from intervention based trials, leading to a paucity of evidence based care.$^{25}$ Adjusting for age, indigenous women are almost twice as likely to have cardiovascular disease compared with non-Indigenous women. $^{7}$

There is a wide range of cardiovascular diseases that can impact pregnancy including: congenital, valvular, cardiomyopathy, ischaemic, arrhythmia and aortic pathology. Of note, there is a significant disparity between the western and developing world. Congenital heart disease accounts for the lead cause of cardiac disease in pregnancy in western countries, contributing approximately $75 \%$ of cases, ${ }^{26}$ whereas in non-western countries, rheumatic heart disease dominates with $60 \%-90 \%$ of cases. ${ }^{22} 27$ These pregnancies are at a higher risk of cardiac, obstetric and neonatal complications compared with their healthy counterparts. One study showed that, on average, maternal complications in women with cardiac disease occurred in nearly $24 \%$ of pregnancies. ${ }^{28}$ Equally as significant, Siu et al reported in their cohort of 562 women with cardiac disease in pregnancy that $20 \%$ had neonatal complications. ${ }^{27}$

One of the most comprehensive studies into this scarcely researched field is the international, multicentre Registry on Pregnancy and Cardiac Disease (ROPAC); however, this was limited in its selection to structural heart disease only. Interestingly, ROPAC found that the highest complication rates occurred in women with pulmonary arterial hypertension. ${ }^{23}$ ROPAC also showed that the following are prepregnancy predictors for mortality and/or heart failure: New York Heart Association class 2 or more, left ventricular ejection fracture $<40 \%$, signs of heart failure and the use of anticoagulants.

\section{Justification for the study}

While perinatal and general disease registries capture some data on pregnancy in women with cardiac and kidney disease, many critical questions about these high-risk pregnancies cannot be answered due to lack of diseasespecific information. Furthermore, there is a paucity of evidence around the current models of care that are in place to support the clinical needs of the women with earlier stages of CKD. A prospective, long-term, cohort study within Australia with a primary purpose of collecting and evaluating the impact of CKD or cardiac disease in pregnancy is an essential step towards addressing this gap in knowledge. This study will be instrumental in enabling women with CKD and/or cardiac disease and their clinicians to effectively prepare, treat and plan for pregnancies based on robust, comprehensive and locally collected data.

This study will explore current outcomes for women, including patient-related outcome measures who have CKD or cardiac disease in pregnancy. It will describe patterns of presentation, pregnancy management, models of care, adequacy of healthcare delivery in these pregnancies over time, the mother's perception and satisfaction of the care they received and any ongoing health issues of concern to them and their children. By monitoring the incidence of maternal and neonatal complications, trends in treatments and performing long-term follow-up of these women, predictors of complications and adverse outcomes will be identified. The unique nature of establishing a combined multidisciplinary kidney and cardiac disease in pregnancy database allows for future collaboration and expansion to include other medical subspecialties. Ultimately, it will be the evidence-based foundation that underpins improvements in pregnancy counselling and care.

\section{METHODS/DESIGN}

\section{Study objectives}

The aim of the study is to prospectively record the incidence, prevalence, aetiology, outcomes and follow-up of maternal CKD and cardiac disease in the obstetric population of South Australia.

Specific objectives are to evaluate:

1. The incidence and impact of known or diagnosed CKD and cardiac disease in pregnancy on obstetric and foetal outcomes.

2. The impact of pregnancy on maternal kidney function and future kidney health.

3. The impact of pregnancy of maternal cardiac function and future cardiovascular health.

4. The current practices and models of care used for management for CKD and cardiac disease in pregnancy.

5 . The impact of CKD or cardiac disease on early ( $<20$ week gestation) pregnancy loss.

\section{Design}

A state-wide, prospective, longitudinal cohort study of women with known acquired or suspected CKD or cardiac disease who are pregnant. It will include women attending public South Australian Hospitals. In 2020, South Australia had appropriately 18800 births which included 4700 at the Women's and Children's Hospital, 3600 at the Lyell McEwin Hospital and 3300 at Flinders Medical Centre. These three metropolitan public obstetric hospitals account for the majority of births in the state. Thirty-six hundred births occur in private hospitals and the remaining 3600 occur in rural South Australia. This longitudinal study will commence recruitment in 2022 and collect data for at least 5 years, with the aim to continue on after this time point for ongoing analyses and refinement.

\section{Participants}

Patient enrolment will occur from existing nephrology, cardiology, obstetric medicine and obstetric clinical services within the public hospitals of South Australia outlined above. In South Australia, the first antenatal visit is generally between 12 and 14 weeks. Complex 
pregnancies from outside of these centres in rural South Australia or private hospitals are referred to these sites as they are tertiary and quaternary centres. Eligible participants will be identified in clinical visits and approached by site clinical staff and given the participant information sheet. Written informed consent will be obtained. Participants for the study will be asked to separately consent to be contacted for any future-related research projects. Non-English speaking patients will be recruited with translator assistance.

\section{Participant selection criteria}

Kidney disease inclusion criteria

We will include all adult women who:

A. Are pregnant and

B. Present with at least one of the following:

a. Preconception diagnosis of CKD of any stage (including dialysis and transplant recipients).

b. Newly identified kidney impairment in pregnancy, defined by any one of the following:

i. Two or more serum creatinine readings above the normal local laboratory reference range for pregnancy.

ii. Significant proteinuria during pregnancy (urine $\mathrm{PCR}>30 \mathrm{mg} / \mathrm{L}$ ), not in the context of pre-eclampsia.

c. Strong clinical suspicion of underlying CKD in women with acute kidney injury or other comorbidity.

Kidney disease exclusion criteria

- Pre-eclampsia with proteinuria, but no other features or clinical suspicion of underlying kidney disease or kidney impairment.

- Uncomplicated hypertension with no other features of kidney disease.

Cardiac disease inclusion criteria

We will include all adult women who:

A. Are pregnant and

B. Present with at least one of the following:

a. Congenital cardiac disease even if fully corrected.

b. Valvular heart disease beyond physiological valvular regurgitation.

c. Cardiomyopathy.

d. Ischaemic heart disease.

e. Arrhythmia.

f. Pulmonary hypertension.

g. Aortic pathology.

Cardiac disease exclusion criteria

- Physiological sinus tachycardia of pregnancy.

- Sinus tachycardia without evidence of other cardiac or autonomic nervous system disease.

- Isolated ectopic beats at rest with normal cardiac structure and function.

General exclusion criteria

- Aged $<18$ years.

- Non-consenting.
- No translator available for recruitment.

Numbers and reasons for exclusion will be recorded.

\section{Assessment of kidney function}

There is a significant alteration of renal physiology during pregnancy that results in an increased GFR with resultant decrease in serum urea and creatinine. ${ }^{29}{ }^{30}$ Causative factors include: an increase in cardiac output and renal blood flow as well as a reduction in serum sodium $(3-5 \mathrm{mmol} / \mathrm{L})$ due to increased intravascular volume. ${ }^{29}$ Renal function in non-pregnant patients is evaluated by calculating the eGFR, with formulae derived from populations using gender, age, ethnicity and serum creatinine measurement. However, eGFR formulae are not valid for use during pregnancy, where serum creatinine usually falls due to renal physiological changes. Thus, eGFR will only be used for prepregnancy creatinine levels and the categorisation of preconception or postpartum CKD. In pregnancy, it is validated that a serum creatinine of $>80-90 \mathrm{mmol} / \mathrm{L}$ usually correlates to a level of renal impairment. ${ }^{31}$ Local laboratory reference ranges will be used. To be included in this study, a formal kidney diagnosis must be made by a nephrologist.

\section{Assessment of cardiac function}

Cardiac physiology is very dynamic during pregnancy and accurate diagnosis of cardiac disease is pregnancy involves multiple non-invasive and occasionally invasive investigations. To be included in this study, a formal cardiac diagnosis must be made by a cardiologist.

\section{Data collection}

Referring clinicians can directly enter the data into the database otherwise, the study project officer will be notified of the case and compile data from patient records at enrolment, the end of each trimester, at birth and 6 weeks post partum. All women will be invited to have follow-up at 6 and 12 months post partum and annually thereafter via a phone call and electronic medical records review by the project officer. Women with multiple pregnancies over the study period will have all eligible pregnancies included.

\section{Summary of collected data}

Common to kidney and cardiac cohorts

- Patient details.

- Previous pregnancies.

- Past medical/obstetric issues.

- Complications in pregnancy.

- Labour and birth.

- Peripartum complications.

- Infant outcomes.

Kidney disease specific data

- Aetiology of kidney disease.

- Management of kidney disease in pregnancy.

- Anti-hypertensive and/or immunosuppressive therapy.

- Renal function during pregnancy. 
- Transplant history.

- Dialysis history and access.

- Kidney function laboratory data.

For kidney disease specific data, the study will use a modified version of the Australasian Maternity Outcomes Surveillance System, ${ }^{31}$ which was previously piloted in in 260 maternity hospitals within Australian for data collection on kidney disease and pregnancy.

Cardiac disease-specific data

- Aetiology of cardiac disease.

- Prior interventions.

- Cardiac devices.

- Cardiac medications.

Postpartum follow-up—common to kidney and cardiac cohorts

- Mortality.

- Patient hospitalisations.

- Infant progress-self-reported by parent.

Major illness/medical diagnosis.

Any hospitalisations.

Developmental issues.

Mortality.

- Blood pressure.

- Current medications.

- Patient-reported outcome measures (PROM).

Postpartum follow-up—-kidney disease-specific data

- Kidney function laboratory data.

- Kidney biopsy since last review.

- Renal replacement therapy since last review.

Postpartum follow-up—cardiac disease-specific data

- Cardiac diagnosis or events since last review.

- Cardiac function laboratory data or imaging.

\section{Models of care}

Using the data collected above, current treatment practices will be determined and then compared with best practice guidelines in subsequent studies.

\section{Study outcomes}

Primary outcomes: incidence and aetiology and outcomes of kidney and cardiac disease in the obstetric population of South Australia.

Secondary outcomes will include:

1. Maternal obstetric outcomes and complications (eg, hypertensive disorders of pregnancy, gestational diabetes mellitus or emergency Cesarian section).

2. Maternal kidney function outcomes including requirement for kidney replacement therapy and postpregnancy CKD stage shift.

3. Maternal cardiac function outcomes including requirement for new medication or medical intervention.

4. Perinatal outcomes including birth outcomes and adverse events (eg, low birth weight, admission to neonatal intensive care or death).

5. Patient perspectives surrounding their chronic disease management during pregnancy.
6. Determining current models of care and evaluating current practice of the medical management of CKD or cardiac disease in pregnancy.

\section{Estimated sample size}

Based on ANZDATA registry data ${ }^{3}$ and local clinician experience, we estimate approximately 20-30 women per year with a pregnancy event with CKD, dialysis or transplant will be eligible within South Australia.

Based on local clinician experience, we estimate approximately 100-200women per year with a pregnancy event with cardiac disease will be eligible within South Australia. For both groups, we anticipate $80 \%$ recruitment rate and $60 \%-80 \%$ retention rate at 1 year post partum.

\section{Estimated timeframe}

Enrolment will commence in January 2022.

\section{Data analysis}

A variety of techniques will be used, as the different hypotheses and data structures require different approaches. Descriptive techniques and multivariate analysis will determine $\mathrm{OR} /$ relative risk of adverse maternal and fetal outcomes, adjusted for maternal demographic and disease-specific parameters. $\chi^{2}$ tests, Student's t-test, Fisher's exact tests and multivariate logistic regression will be used as appropriate. Analyses will be performed using Stata V.16.0.

\section{Ethics and dissemination}

This research proposal will be conducted in accordance with the National Statement on Ethical Conduct in Human Research 2007. Approval (2021/HRE00344) was obtained from the local Health Network Human Research Ethics Committee involved in the study. All participants can withdraw from the study at any time without fear of jeopardising current or future medical treatment. The participant must email the study project officer with their intent to withdrawal from the study and all data related to that individual will be deleted.

\section{Patient and public involvement}

This project has been codesigned with a consumer reference group, established in 2019, who have been working with the investigator team to develop the study concept and plan. They have had input into the study protocol, data collection tool and research objectives.

\section{Data storage}

Data for this study will be stored on a password-protected database managed by REDCap in line with ICH Good Clinical Practice for researchers. REDCap has a secure server, used by researchers globally for management of databases and it also protects against loss of data, as the database will be accessible from any computer. The data will be collected directly from the case notes and transferred immediately to the database spreadsheet. Patient information on REDCap will be deidentified and allocated a non-identifiable study ID. A master list 
with a patient's medical record number and their allocated study ID number will be on an excel spreadsheet, securely stored on the personal computer of one of the study project officers.

\section{Dissemination and data reporting}

Summarised data from this study will be presented in an annual report. These findings will be published in peerreviewed journals and at suitable national and international meetings. It will be reported in accordance with the Strengthening the Reporting of Observational Studies in Epidemiology reporting criteria for cohort studies. ${ }^{32}$ Furthermore, findings will be presented to relevant stakeholders (clinicians and policy makers) to maximise translational impact of research findings. Additionally, consumer versions of research outputs will be developed in conjunction with the consumer reference group.

\section{DISCUSSION}

It is anticipated that the results of this cohort study will identify the majority of women within the South Australian community affected by CKD or cardiac disease in pregnancy. This will allow an understanding of the incidence and prevalence of these important comorbidities. This study will uniquely evaluate early stages of CKD in pregnancy, which currently has very limited literature or data capture in existing renal datasets. In regards to cardiac disease in pregnancy, this study is evaluating and including a broad aetiology including arrhythmia which has infrequently been researched before.

There is significant collaboration within the state and referrals to tertiary and quaternary centres are common practice. Obstetric physicians, and physicians with special interests in obstetric medicine service these multiple centres and thus, a simple recruitment process and good endorsement of the study is expected. The REDCAP database is an excellent tool that will allow prompt analysis of results and allows multiple data entry points overtime which is critical for this study over the course of the pregnancy.

This study will highlight the risks associated with CKD and cardiac disease during pregnancy and will act as a catalyst for further investigations towards intervention and management. There is a critical paucity of information and proposed models of care for these high-risk chronic conditions in pregnancy. Data on outcomes and risk factors for adverse outcomes will be used in local health services and more broadly to inform improvements in patient safety and quality of care. The data will inform frameworks for prepregnancy counselling, training to health professions and ensuring appropriate postpartum follow-up. The study will specifically include PROMs to ensure that outcomes are inclusive of patient input. This new knowledge generation will be shared with consumer partners and used to educate, inform and counsel women on the impact of kidney and cardiac disease in pregnancy. The end-point is to facilitate shared decision through resources for clinicians, patients and their family outlining the implications of their chronic disease on their pregnancy.

Identification of CKD and cardiac disease in pregnancy allows for optimal management and improved outcomes throughout the pregnancy and potentially the rest of women's life. This study will provide valuable information on the real-world trends which will help support decisionmaking for future intervention to improve health outcomes. It is expected to be of significant interest to clinicians and researchers involved in obstetric medicine around the world, but most importantly aligns to the patient priority of achieving motherhood safely.

This study has several key strengths. First, it is the only obstetric study in Australia to collaborate and share a common set of patient variables and then collect disease specific data. The multidisciplinary nature of this study means it will capture a large cohort of medically complex obstetric patients. The prospective cohort design will allow the research team to follow the patients' progress over the 12 months post partum, as well as conduct follow-up of infants. In particular, the ability to follow the impact of pregnancy on future kidney and cardiac function is a unique component of this study.

One of the limitations of this study is that some early forms of CKD are not symptomatic nor have biochemical changes thus will not be detected. This is particularly true for CKD which includes morphological alterations of kidney parenchyma, such as polycystic kidney disease. In particular, the differentiation between pre-eclampsia and CKD is difficult however this area will be evaluated in future studies. Challenges of a comphrensive cohort study include the burden of accurate, prospective and complete data collection. This will be overcome by employing a data entry officer paid for through grant funding. Recruitment bias is possible, as women must provide written informed consent to participate in the study. It is acknowledged that patients captured in this study will be receiving care in public institutions only; however, the majority of complex disease in pregnancy is managed exclusively in state-run hospitals.

\section{Future plans}

Once successfully established in South Australian centres, a key aim is to extend this cohort study to other jurisdictions, and there is already interest from interstate centres to participate. There is potential to develop this into a national ongoing prospective cohort study or registry which will be an enormous step forward for the field of obstetric cardiology and nephrology. Sharing of this data across national and international boundaries will overcome the constant limitation of low event rates and poor statistical power, which has plagued research efforts in the past. Furthermore, the unique nature of establishing a combined multidisciplinary kidney and cardiac disease in pregnancy database allows for future collaboration and expansion to include other uncommon medical conditions that may affect pregnant cohorts. 
Contributors All authors collaborated on the design and research questions. JH, $\mathrm{EH}$ and EA completed the first draft of the manuscript. All authors reviewed the manuscript and provided further input to the final draft.

Funding The authors have not declared a specific grant for this research from any funding agency in the public, commercial or not-for-profit sectors.

Competing interests None declared.

Patient and public involvement Patients and/or the public were involved in the design, or conduct, or reporting, or dissemination plans of this research. Refer to the Methods section for further details.

Patient consent for publication Consent obtained directly from patient(s) Provenance and peer review Not commissioned; externally peer reviewed.

Open access This is an open access article distributed in accordance with the Creative Commons Attribution Non Commercial (CC BY-NC 4.0) license, which permits others to distribute, remix, adapt, build upon this work non-commercially, and license their derivative works on different terms, provided the original work is properly cited, appropriate credit is given, any changes made indicated, and the use is non-commercial. See: http://creativecommons.org/licenses/by-nc/4.0/.

ORCID iD

Jarrad Hopkins http://orcid.org/0000-0002-2913-3312

\section{REFERENCES}

1 Webster P, Lightstone L, McKay DB, et al. Pregnancy in chronic kidney disease and kidney transplantation. Kidney Int 2017;91:1047-56.

2 Piccoli GB, Cabiddu G, Attini R, et al. Risk of adverse pregnancy outcomes in women with CKD. JASN 2015;26:2011-22.

3 Australia and New Zealand dialysis and transplant registry, 42nd annual report, 2019. Available: https://www.anzdata.org.au/report/ anzdata-42nd-annual-report-2019/ [Accessed 29 Jan 2020].

4 Australian Institute of Health and Welfare. Cardiovascular disease, diabetes and chronic kidney disease-Australian facts: prevalence and incidence. cardiovascular, diabetes and chronic kidney disease series No. 2. cat. No. CDK 2. Canberra: AlHW, 2014.

5 kidney health Australia. Chronic kidney disease (CKD) management in primary care. 4 edn. Melbourne, Australia, 2020.

6 Australian Institute of Health and Welfare. Chronic kidney disease Cat. no. CDK 16. Canberra: AIHW, 2019. Available: https://www. aihw.gov.au/reports/chronic-kidney-disease/chronic-kidney-disease [Accessed 22 Apr 2020].

7 Australian Institute of health and welfare. Australian burden of disease study: impact and causes of illness and death in Australia 2015. In: Australian burden of disease series no. 19. Cat. no. BOD 22. 25. Canberra: AlHW, 2019.

8 Fitzpatrick A, Mohammadi F, Jesudason S. Managing pregnancy in chronic kidney disease: improving outcomes for mother and baby. Int $J$ Womens Health 2016;8:273-85.

9 Hewawasam E, Davies CE, Gulyani A, et al. Factors influencing fertility rates in Australian women receiving kidney replacement therapy: analysis of linked Australia and New Zealand Dialysis and Transplant Registry and perinatal data over 22 years. Nephrology Dialysis Transplantation 2021;57e.
10 Munkhaugen J, Lydersen S, Romundstad PR, et al. Kidney function and future risk for adverse pregnancy outcomes: a populationbased study from HUNT II, Norway. Nephrol Dial Transplant 2009;24:3744-50.

11 Cabiddu G, Castellino S, Gernone G, et al. A best practice position statement on pregnancy in chronic kidney disease: the Italian Study Group on kidney and pregnancy. J Nephrol 2016;29:277-303.

12 Zhang J-J, Ma X-X, Hao L, et al. A systematic review and metaanalysis of outcomes of pregnancy in CKD and CKD outcomes in pregnancy. Clin J Am Soc Nephrol 2015;10:1964-78.

13 Fitzpatrick A, Venugopal K, Scheil W, et al. The spectrum of adverse pregnancy outcomes based on kidney disease diagnoses: a 20-year population study. Am J Nephrol 2019;49:400-9.

14 Hilder L, Zhichao Z, Parker M. Australia's mothers and babies 2012. Perinatal statistics. Canberra: AlHW, 2014.

15 Bramham K, Lightstone L. Pre-pregnancy counseling for women with chronic kidney disease. J Nephrol 2012;25:450-9.

16 Jesudason S, Grace BS, McDonald SP. Pregnancy outcomes according to dialysis commencing before or after conception in women with ESRD. Clin J Am Soc Nephrol 2014;9:143-9.

17 Piccoli GB, Cabiddu G, Attini R, et al. Risk of adverse pregnancy outcomes in women with CKD. J Am Soc Nephrol 2015;26:2011-22.

18 Jones DC, Hayslett JP. Outcome of pregnancy in women with moderate or severe renal insufficiency. $N$ Engl J Med 1996;335:226-32.

19 McDonald SD, Malinowski A, Zhou Q, et al. Cardiovascular sequelae of preeclampsia/eclampsia: a systematic review and meta-analyses. Am Heart J 2008;156:918-30.

20 Garcia M, Mulvagh SL, Merz CNB, et al. Cardiovascular disease in women: clinical perspectives. Circ Res 2016;118:1273-93.

21 Regitz-Zagrosek V, Roos-Hesselink JW, Bauersachs J, et al. 2018 ESC guidelines for the management of cardiovascular diseases during pregnancy. Eur Heart J 2018;39:3165-241.

22 Gandhi M, Martin SR. Cardiac disease in pregnancy. Obstet Gynecol Clin North Am 2015;42:315-33.

23 Roos-Hesselink J, Baris L, Johnson M, et al. Pregnancy outcomes in women with cardiovascular disease: evolving trends over 10 years in the ESC registry of pregnancy and cardiac disease (ROPAC). Eur Heart J 2019;40:3848-55.

24 Management of cardiac conditions in pregnancy. Cardiol Today 2016;6:14-20.

25 Arnott C, Patel S, Hyett J, et al. Women and cardiovascular disease: pregnancy, the forgotten risk factor. Heart Lung Circ 2020;29:662-7.

26 Swan L. Congenital heart disease in pregnancy. Best Pract Res Clin Obstet Gynaecol 2014;28:495-506.

27 Siu SC, Sermer M, Colman JM, et al. Prospective multicenter study of pregnancy outcomes in women with heart disease. Circulation 2001;104:515-21.

28 Goldstein SA, Ward CC. Congenital and acquired valvular heart disease in pregnancy. Curr Cardiol Rep 2017;19:1-7.

29 Tran HA. Abnormal laboratory results: biochemical tests in pregnancy. Aust Prescr 2005;28:98-101.

30 Hytten F. Blood volume changes in normal pregnancy. Obstet Gynecol Surv 1986;41:426-8.

31 Safi N, Sullivan E, Li Z, et al. Serious kidney disease in pregnancy: an Australian national cohort study protocol. BMC Nephrol 2019;20:230.

32 von Elm E, Altman DG, Egger M, et al. The strengthening the reporting of observational studies in epidemiology (STROBE) statement: guidelines for reporting observational studies. J Clin Epidemiol 2008;61:344-9. 